\title{
The unified phenomenological model of light curves of stellar flares
}

\author{
H. M. Tovmassian ${ }^{1}$, V. P. Zalinian ${ }^{2}$, N. A. Silant'ev ${ }^{1, \star}$, O. Cardona $^{1}$, and M. Chavez ${ }^{1}$ \\ ${ }^{1}$ Instituto Nacional de Astrofísica Optica y Electrónica, Apartado Postal 51 y 216, CP 72000, Puebla, Pue., México \\ e-mail: hrant@inaoep.mx; silant@inaoep.mx; ocardona@inaoep.mx;mchavez@inaoep.mx \\ 2 V.A. Ambartsumian Byurakan Astrophysical Observatory, NAS, Armenia \\ e-mail: zalinian@bao.sci.am
}

Received 15 April 2002 / Accepted 25 November 2002

\begin{abstract}
We present a phenomenological model to explain a wide variety of stellar flares. We assume that a flare consists of a fast and relatively strong rise in brightness followed by a slow and fainter component. The latter is a result of re-radiation of the part of the energy of the prime flare by the photosphere of the star. The model is based primarily on geometrical consideration in which the position of the flare with respect to the stellar observable disk gives rise to different flare morphologies. In general, flares near the center of the disk consist of a very small pre-flare dimming. When the flare occurs towards the edge of the stellar disk the pre-flare dimming becomes fainter and eventually unobservable, while the re-radiated component also becomes fainter and redder. When the flare takes place on the backside of the stellar surface as seen by us, but near the limb, we may see only the re-radiated component.
\end{abstract}

Key words. stars: general

\section{Introduction}

On the basis of a statistical study of flare stars in the young cluster Pleiades it was concluded that almost all faint stars of this cluster are flare stars, and that the phenomenon of flare activity is very common, or always present in the early phases of evolution (Ambartsumian 1969; Mirzoyan et al. 1981). However, this fact is almost completely ignored in star formation theories. Yet the flare event may be very important for the understanding of stellar formation and early evolution. During stellar flares the energy release can be as high as $3 \times 10^{36}$ which is almost five orders of magnitude of that released during a solar flare (Gershberg 1989; Shakhovskaya 1989).

The wide variety the light curve forms complicates the understanding of the mechanism(s) of flaring. Inspection of the light curves of flares registered with a high time resolution shows that the multitude of different manifestations may roughly be represented by the following types. First there is a fast increase of brightness that lasts a few seconds followed by a relatively slow decline (Fig. 1), but composite flares are observed that consist of two or more sharp brightness peaks. Very often the decline is followed by a second, fainter and much slower component (Fig. 2), but in some cases only a slow rise and slow decline of brightness is observed (Fig. 3). In a few cases a dimming of the star precedes the flare that is, however,

Send offprint requests to: $\mathrm{H}$. M. Tovmassian,

e-mail: hrant@inaoep.mx

* Permanent adress: Pulkovo Astronomical Observatory,

St. Petersburg, Russia.

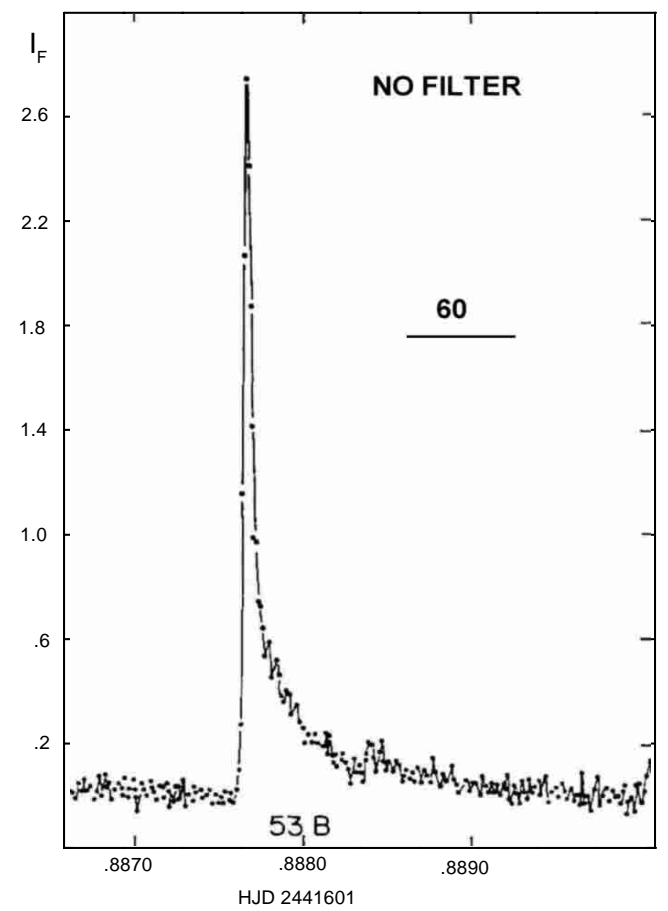

Fig. 1. The flare observed on UV Ceti (Moffett 1974b).

so small that it may not always be observed (Fig. 4). Huge energies may be released in spiky flares lasting less than a few tenths of a second (Tovmassian \& Zalinian 1988; Tovmassian et al. 1997b). 
In order to explain the color variations of flares during the decrease of brightness Kunkel (1967) proposed a twocomponent model of flares, in which the second component originates as the result of a heating of the photosphere by the prime flare farther out in the atmosphere. High time-resolution observations of flares in recent years allow us to separate accurately the various components, and to suggest a unified phenomenological model of the light curves. In this paper we argue that generally the flare consists of a sharp rise of brightness and an exponential decline. We show that the slow component, in accordance with Kunkel's model (1967), is re-radiated emission of the prime flare by the star photosphere. Depending on the locations of the flare on the visible disk of the star we observe different manifestations of light curves.

\section{The model}

We propose a unified phenomenological model of flares according to which a multitude of light curves of flares can be explained by a projection effect.

The flare occurs at high levels of the chromosphere or above it and illuminates partially the photosphere. The size of the illuminated area depends on the height of the flare above the photosphere. The optical depth of the photosphere for elastic (Thompson) scattering in these cool $\mathrm{K}$ and $\mathrm{M}$ type stars is sufficiently small and practically all infalling emission is absorbed and scattered by the photosphere. As a result of the ionization and recombination processes, the absorbed shortwave emission is distributed at longer wavelengths. The absorbed emission finally is re-radiated out from the photosphere and is observed as a second component of the flare. Hence, such flares are not superposition of independent flares, as has been supposed by Kilyachkov et al. (1979), Mirzoyan (1980), Abdul-Aziz et al. (1995), but rather second, slow re-emission of the main flare. We call the second component the echo of the primary flare.

Grinin (1973) was the first to consider the transfer problem for nonstationary radiation in a grey atmosphere disturbed by the radiation of the flare. More recently Kolesov \& Sobolev (1990) estimated the average time delay of re-radiation for a hydrogen photosphere. They showed that the delay time depends on the photospheric temperature and on the hydrogen density. For $\mathrm{K}$ and $\mathrm{M}$ stars the re-radiation time is $\sim 30 \mathrm{~s}$ at hydrogen densities of about $n_{\mathrm{H}}=10^{14} \mathrm{~cm}^{-3}$, and is about $\sim 300 \mathrm{~s}$ at $n_{\mathrm{H}} \sim 10^{16} \mathrm{~cm}^{-3}$. Apparently, the deduced values of time delays are approximate, since Kolesov \& Sobolev (1990) considered a simplified uniform photosphere model, and the process of diffusion of photons was considered in the rough Eddingtonian two-streams approximation.

The analysis of flares observed with high time resolution allows us to separate accurately the different components, and to investigate the possible correlations between different parameters. Decomposition of light curves was done assuming that the slow decline of the prime flare may be approximated as "proportional to" $e^{-\beta t}$ (Chugainov 1962). An example of decomposition is shown in Fig. 5. If the time resolution is not high enough, e.g. it is larger than a second, it becomes impossible to separate accurately both components.
Table 1. The parameters of two component flares of V577 Mon.

\begin{tabular}{llllll}
\hline \hline$\Delta U_{1}$ & $\begin{array}{l}\Delta U_{2} \\
{[\mathrm{mag}]}\end{array}$ & $\begin{array}{l}(U-B)_{1} \\
{[\mathrm{mag}]}\end{array}$ & $\begin{array}{l}(U-B)_{2} \\
{[\mathrm{mag}]}\end{array}$ & $\begin{array}{l}\Delta t \\
{[\mathrm{mag}]}\end{array}$ & $\mathrm{s}$ \\
\hline 1.0 & 0.4 & 0.6 & 1.0 & 70 & \\
& 1.5 & 0.4 & 0.2 & 0.9 & 50 \\
1.1 & 0.4 & 0.2 & 1.0 & 60 & \\
0.4 & 0.2 & 0.8 & 1.1 & 150 & \\
2.5 & 1.3 & -0.4 & 0.0 & 40 & \\
\hline
\end{tabular}

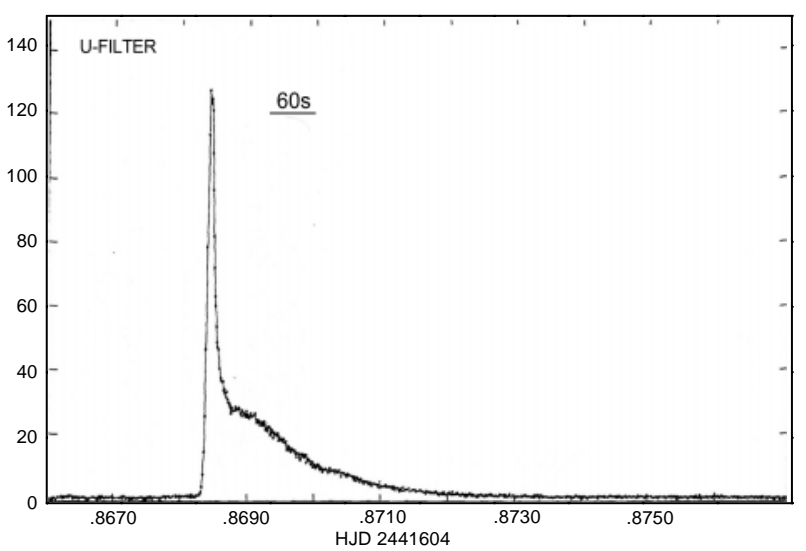

Fig. 2. The flare observed on UV Ceti by Bopp \& Moffett 1973.

We have decomposed five flares of V577 Mon observed with $0.1 \mathrm{~s}$ time resolution (Tovmassian et al. 1997a; Zalinian et al. 2002b) and determined the parameters of two components listed in Table 1. In Cols. 1, 2, 3 and 4 the amplitudes $\Delta U_{1}, \Delta U_{2}$, and the colours $(U-B)_{1},(U-B)_{2}$ of the prime and of the second (the echo) components is given respectively. The time delay between the two components are given in Col. 5 . Note that the time delay between the prime flare and the secondary peak is within 40-150 s, in good agreement with the estimate by Kolesov \& Sobolev (1990).

In previous works (Tovmassian \& Zalinian 1988; Tovmassian et al. 1997b) we have shown that the colour $U-B$ is well correlated with the maximum intensity $\Delta U_{1}$ : bluer colours for increasing amplitudes. In Fig. 6 we show the diagram $\Delta U_{1}$ versus $(U-B)$ for the stars EV Lac and V577 Mon (Tovmassian et al. 1997b; Zalinian et al. 2002a; Zalinian et al. 2002b). The lines plotted in Fig. 6 correspond to a linear regression for EV Lac (solid line) and for V577 Mon (dashed line). In both cases the rms is small $(\approx 0.05)$ with a slightly redder $\approx 0^{\mathrm{m}} .25$ correlation for V577 Mon.

Butler et al. (1981), Byrne \& McKay (1990), Mathioudakis \& Doyle (1990) have given evidence that most part of the flare energy is in the far UV (below $300 \mathrm{~nm}$ ) or even in the X-ray part of the spectrum. This implies that the larger the difference between the wavelength of observation and the wavelength of the maximum of emission, the smaller will be the measured flux magnitude (in our case: $\Delta U$ ) and the longer will be the time delay. In Fig. 7 we display the amplitudes $\Delta U_{1}$ of the prime flares of V577 Mon (estimated with an accuracy of $\approx 0$. 05 ) as a function of the time lag between the main flares and the echoes. In spite of a small statistics (we have only five points), it is seen that, as expected, the time lag decreases with the increase of the 


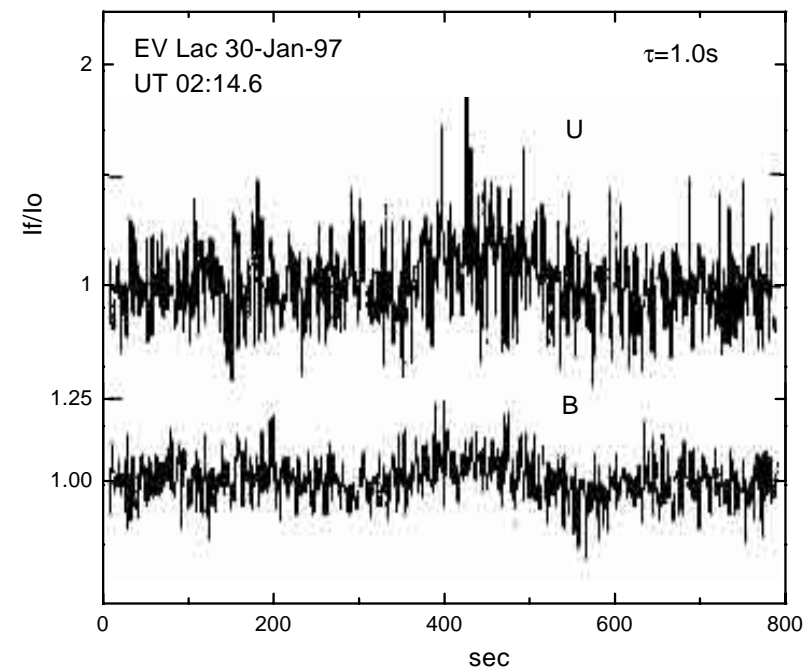

Fig. 3. The flare observed on EV Lac (Tovmassian et al. 1997b).

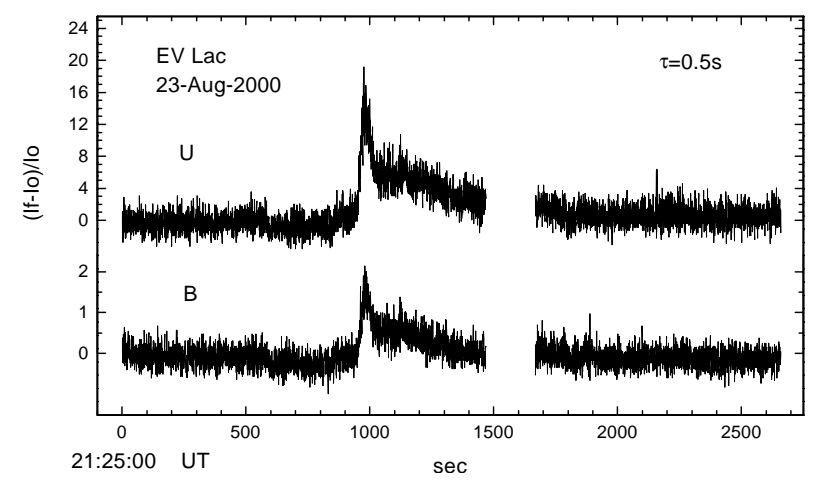

Fig. 4. The flare of EV Lac observed on 23 August 2000 (Zalinian et al. 2002a).

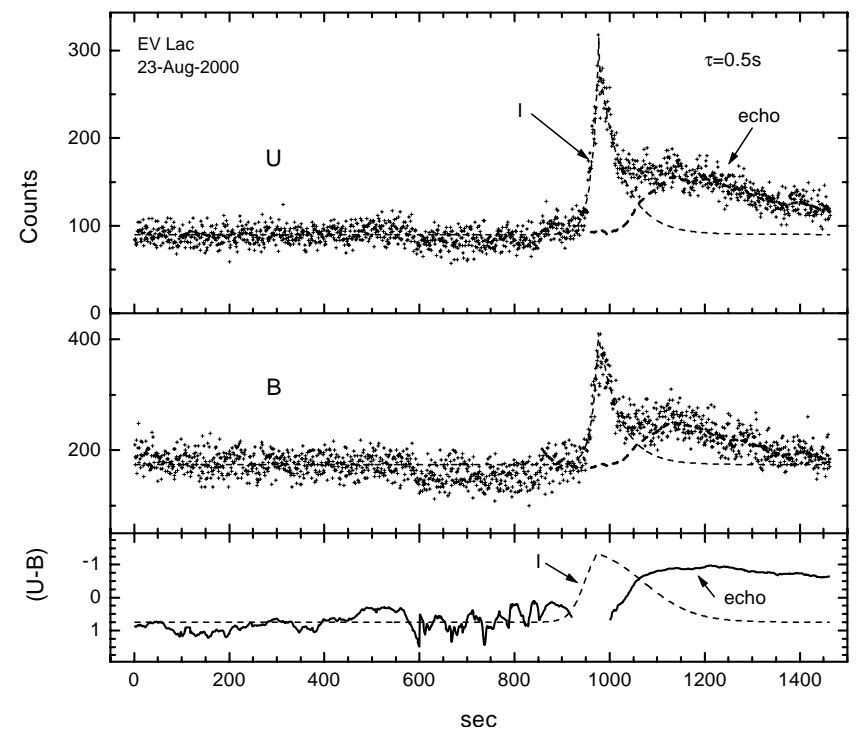

Fig. 5. Decomposition of the flare of EV Lac observed on 23 August 2000 (Zalinian et al. 2002a).

brightness of the flare in $U$ filter. Certainly more observations are required to determine unambiguously the above correlation.

In general the amplitude of the echo is rising and declining very slowly. The release of the energy takes, on average, several

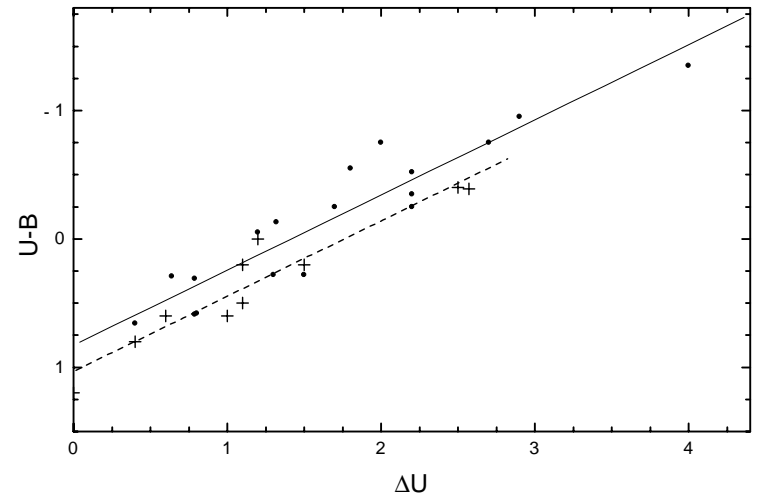

Fig. 6. The dependence of the colour $U-B$ of the flare on the amplitude $\Delta U$ for EV Lac (dot) and V577 Mon (+).

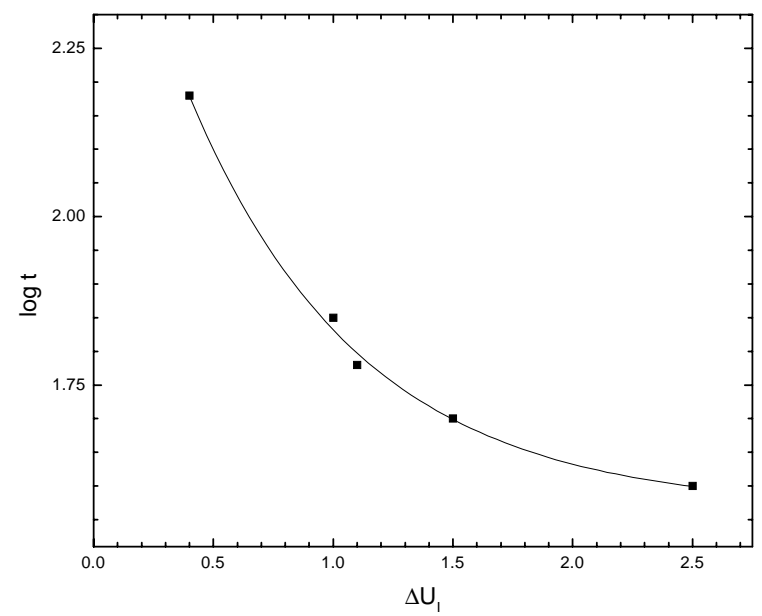

Fig. 7. The dependence of the time delay $t$ between the prime flare and the echo on the amplitude of the prime flare $\Delta U$ for V577 Mon.

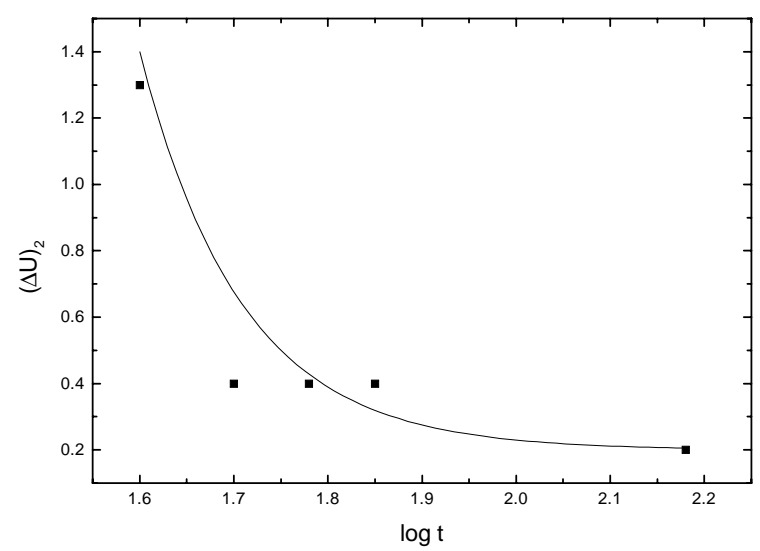

Fig. 8. The dependence of the amplitude $\Delta U_{2}$ of the echo on the time delay $t$ for V577 Mon.

minutes. The re-radiated emission is very probably redder than the primary flare. As indicated in Table 1 (Cols. 3 and 4), the colours of the echo are indeed systematically redder than those of the primary. Aditionally, the amplitude $\Delta U_{2}$ of the echo appears well correlated with the time delay between the primary and the echo (Fig. 8). Indeed, the more is the time delay between the prime flare and the re-radiated emission, the fainter the latter should be. 


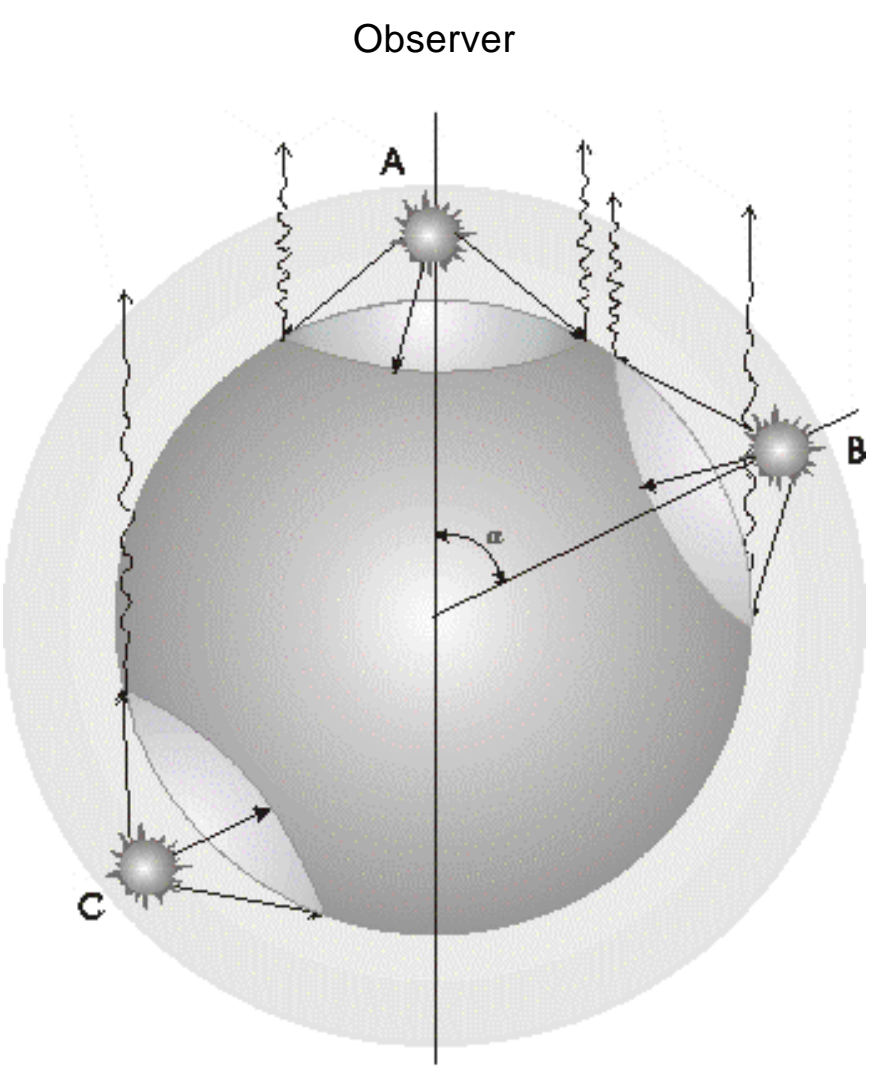

Fig. 9. The scheme of flares at different locations on the surface of the flare star.

The dimming before a flare and the reddening may be interpreted by the presence of a cool spot on the visible stellar disk. In the case of the flare shown in Fig. 4 the reddening by 0 . 15 lasts for about $13 \mathrm{~min}$. Such reddening would be explained by decrease of the temperature of the whole visible photosphere from $4300 \mathrm{~K}$ to $4000 \mathrm{~K}$. If the spot is of smaller size, say, it covers only $40 \%$ of the disk, the temperature of the spot needed for the observed dimming should be $3600 \mathrm{~K}$. If we are dealing with smaller spots, even a lower temperature would be required, however, and the color of the star would no longer change. The dimming time is extremely short in comparison to the life time of the solar spots. Therefore, if dimming is due to a starspot, its size and life time certainly differ from that of the solar spots.

From the considerations described above we can now use our model to interpret the light curves displayed in Figs. 1-4. In Fig. 9 we illustrate a schematic diagram of the stellar surface with three representative points.

The light curve starts with a small decrease of brightness of the star for a few hundred seconds followed by a fast increase and decrease of brightness, and next by a slow component (Fig. 4). We observe these brightness curves, if the flare occurs near the center of the visible disk of the flare star (position $A$ in Fig. 9). If the flare occurs at some angular distance $\alpha$ from the center of the visible disk of the star (for instance at position $B$ in Fig. 9), the dimming will be hard to detect, since it is so small (see Fig. 4 in which a relatively strong dimming is observed). At $\alpha \approx 45^{\circ}$ the projected area of the cool spot is decreased by $\approx 0.7$. The echo of the flare will then also be fainter,

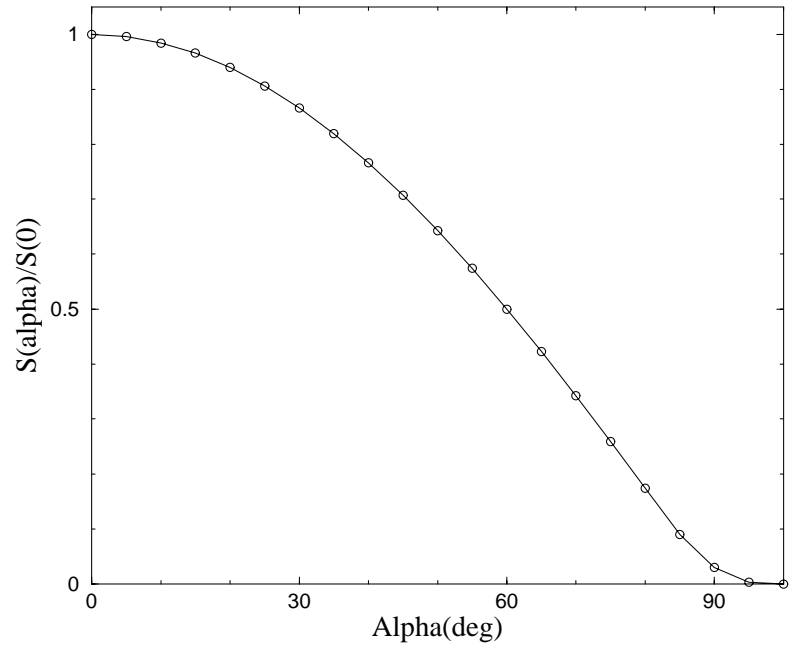

Fig. 10. The dependence of the area of the observed surface of "echo" on the angular distance $\alpha$ from the center of the star disk.

because the re-radiated emission is observed in this case from a smaller area of the photosphere due to the projection effect. Figure 10 illustrates the decrease of the normalized area of the projection of the illuminated spot over sky with increase of the angle $\alpha$, and it is in this projected spot that determines our observation. Calculations were done assuming that the ratio $h / R$, where $h$ is the hight of the flare event above the photosphere of the dwarf $M$ type star with radius $R$ is about 0.01 (Falchi \& Mauas 1998; Fawzy et al. 2002). The assumption that the $h / R$ ratio is equal to 0.005 or 0.020 does not change the rate of decrease of the area of the projection of the illuminated spot over the sky with the increase of $\alpha$.

If a flare occurs at not very high angular distance $\alpha$ from the center of the observed hemisphere, we observe a light curve presented in Fig. 2. The farther from the center of the star disk the flare occurs, the fainter is the echo. In flares that occur near the limb and somewhat beyond it, (position $C$ in Fig. 9), the echo may not be seen at all (Fig. 1). If a powerful flare occurs at the hidden part of the surface of the star, we obviously do not see it, but may observe a faint echo diffused through the photosphere (Fig. 3). The brightness of such flare rises and declines very slowly. Meanwhile, the amplitude, colour and dynamics of the first, fast flare do not depend on the position on the disk.

The rotation period of red dwarfs is much longer than the time delay of re-radiated emission. Therefore, even in the case when the rotation axis of the star is orthogonal to the line of sight, the rotation of the star would not alter the light curve of the echo.

The surface of the visible hemisphere where only half of the illuminated spot is seen is about 0.3 of that of the whole hemisphere. It means that the number of flares with very faint echo, i.e. consisting only of the sharp rise and decline in brightness must be about three times smaller than those of flares with significant echo emission. 


\section{Conclusions}

Consideration of different types of flares allow us to propose the following scenario of flares. A flare occurs in the area of the formed starspot. The energy of the flare emitted downward is absorbed in the photosphere and after a diffusion in space and frequency is re-radiated. The second component of the flare, the so-called echo is formed as the result of re-radiation of the flare emission by the photosphere. The multitude of different types of light curves may be explained by a geometrical effect. The typical flare light curve begins with a weak dimming of the brightness of the star for a few minutes, followed by the primary, fast flare, and by a second component, the echo. Depending on the location of the flare on the stellar surface with respect to the line-of-sight we observe its different manifestations. If the flare occurs near the center of the disk of the star, the dimming of the star brightness is observed. The echo is relatively bright. If the flare occurs at some angular distance from the center of the star disk, the dimming may not be observed, and the echo is fainter. A flare that occurred near the limb would not have measurable echo. We may see only the echo, if the prime flare occurred at the hidden side of the star, not far from the limb. A theory of the flare event must explain only the main flare with its very sharp rise of the brightness and total duration of a few seconds or tens of seconds.

Haisch et al. (1991) mention that in spite of a lot of observational data accumulated on flare stars "no model has been able to describe all aspects, nor do we have a reliable and comprehensive energy budget". García-Alvarez et al. (2002) wrote recently that "the exact mechanism(s) leading to the energy release and subsequent excitation of various emission features remains poorly understood". The simplified phenomenology of flares presented in this paper may help to solve the problem of the flare event.

Acknowledgements. We are grateful to the anonymous referee for valuable comments that allowed us to improve the paper significantly, and to Dr. Habing for careful reading of the paper and its substantial improvment. VPZ is grateful to the CONACYT Project34564-E and to the INAOE for the financial support and hospitality during thereduction of observational data and preparation of paper at INAOE.

\section{References}

Abdul-Aziz, H., Abranin, E. P., Alekseev, L. Yu., et al. 1995, A\&AS, 114,509

Ambartsumian, V. A. 1969, in Stars, Nebulae, Galaxies, ed. L. V. Mirzoyan (Academy Sci. Armenian SSR)

Bopp, B. W., \& Moffett, T. J. 1973, ApJ, 185, 239

Butler, C. J., Byrne, P. B., Andrews, A. D., \& Doyle, J. G. 1981, MNRAS, 197, 215

Chugainov, P. F. 1962, Izv. Crimean Obs., 28, 150

Chugainov, P. F. 1965, Izv. Crimean Obs., 33, 215

Deming, D., \& Webber, J. C. 1974, IBVS, 672

Falchi, A., \& Mauas, J. D. 1998, A\&A, 336,281

Fawzy, D., Ulmschneider, P., Stępien, K., Musielak, Z. E., \& Rammacher, W. 2002, A\&A, 386, 983

García-Alvarez, D., Jevremović, D., Doyle, J. G., \& Butler, C. J. 2002, A\&A, 383, 548

Gershberg, R. E. 1989, Mem. Soc. Astron. Ital., 60, 263

Grinin, V. P. 1973, Izvestia Cremean Obs., 48, 58

Haisch, B. M., Strong, K. T., \& Rodonó, M. 1991, ARA\&A, 29, 275

Kilyachkov, N. N., Melikian, N. D., Mirzoyan, L. V., \& Shevchenko, V. S. 1979, Astrofizika, 15, 605

Kolesov, A. K., \& Sobolev, V. V. 1990, Sov. Astron., 34, 179

Kunkel, W. 1967, AJ, 72, 810

Mathioudakis, M., \& Doyle, J. G. 1990, A\&A, 232, 114

Mirzoyan, L. V. 1980, in Flare Stars, Fuors and Herbig-Haro Objects, ed. L. V. Mirzoyan (Armenian Acad. Sci. Press), 45

Moffett, T. J. 1974, Sky \& Telescope, 48, 94

Sanwal, B. B. 1974, IBVS, 932

Sanwal, B. B. 1976, IBVS, 1180

Shakhovskaya, N. I. 1989, in Solar and Stellar Flares, ed. B. Haisch \& Rodonó (Kluwer Academic Publisher), IAU Coll., 104, 375

Tovmassian, H. M., \& Zalinian, V. P. 1988, Astrofisika, 28, 131

Tovmassian, H. M., Recillas, E., Cardona, O., \& Zalinian, V. P. 1997a, IBVS, 4465

Tovmassian, H. M., Recillas, E., Cardona, O., \& Zalinian, V. P. 1997b, Rev. Mex. A\&A, 33, 107

Zalinian, V. P., \& Tovmassian, H. M. 1987, IBVS, 2992

Zalinian, V. P., \& Tovmassian, H. M. 1997, IBVS, 4464

Zalinian, V. P., Tovmassian, H. M., \& Cardona, O. 2002a, IBVS, 5256

Zalinian, V. P., Tovmassian, H. M., Cardona, O., \& Chavez, M. 2002b, IBVS, 5272 